\title{
A Distributed Transaction Mechanism for Electricity Market with Electric Vehicles and Blockchain
}

\author{
Zhaoxiong Huang ${ }^{1}$, Dashen Chen ${ }^{1}$, Chun Sing Lai ${ }^{2}$, Zhuoli Zhao ${ }^{1,{ }^{*}}$, Loi Lei Lai ${ }^{1,{ }^{*}}$, Minghao Wang ${ }^{3}$ \\ ${ }^{1}$ School of Automation, Guangdong University of Technology, Guangzhou 510006, China \\ ${ }^{2}$ Department of Electronic \& Computer Engineering, Brunel University London, London, UB8 3PH, UK \\ ${ }^{3}$ Department of Electrical Engineering, The Hong Kong Polytechnic University, Kowloon, Hong Kong \\ E-mail: zhuoli.zhao@gdut.edu.cn, 1.1.1ai@gdut.edu.cn
}

\begin{abstract}
As distributed transactions become more and more mature, such as peer-to-peer (P2P), the widespread application of distributed transactions not only requires fair and open transaction process, but also has as little impact on the main network as possible. This paper considers an energy matching model, which includes two parts. The first part is a kind of new auction, in which participants can clearly aware of competitive pressures because of current or historical data in blockchain. The second part is an interaction between charging station operator and unmatched participants in the first part. Cases have been studied to demonstrate the effectiveness of this distributed transaction.
\end{abstract}

Index Terms-Blockchain, Energy matching model, Electricity market, Electric vehicles

\section{NOMENCLATURE}

Variables and Functions:

$\begin{array}{cl}e, f & \text { Rank expectation of buyer } \\ v_{i}^{b} & \text { Evaluation price of buyer } i \\ b_{i} & \text { Bidding price of buyer } i \\ W_{i}^{\mathrm{b}} & \text { Bidding quantity of buyer } i \\ P & \text { Probability function } \\ \phi & \text { Bidding strategy function } \\ \operatorname{Pr}_{i, t}^{b} & \text { Possibility of EV buyers working with CSO } \\ \operatorname{Pr}_{j, t}^{s} & \text { Possibility of EV sellers working with CSO } \\ P_{t}^{c}, P_{t}^{d} & \text { The charge and discharge amount of CSO } \\ E^{b} & \text { energy storage system at time } t \\ & \text { Mathematical expectation of the transaction } \\ F & \text { volume when participants are partially matched } \\ C & \text { in double auction } \\ \delta & \text { The objective function of CSO } \\ W_{i, t}^{b, C S O} & \text { The social welfare function of CSO } \\ W_{j, t}^{s, C S O} & \text { The transaction amount that CSO trade with the } \\ u_{t}^{+}, u_{t}^{-} & \text {The transaction amount that CSO trade with the } \\ & \text { Auxiliary variable }\end{array}$

Constants and Sets:

$O \quad$ Breakpoint in double auction mechanism

$o \quad$ The value of the breakpoint $a$ on the $\mathrm{x}$ axis

$b^{\text {CSO }} \quad$ Price of CSO for trading with buyers

${ }_{s}$ CSO Price of CSO for trading with sellers

$\lambda^{G} \quad$ Cost of purchasing electricity from the grid for the CSO

\begin{tabular}{|c|c|}
\hline Num_B & Number of buyers \\
\hline Num_S & Number of sellers \\
\hline Num_T & Number of time slot \\
\hline $\operatorname{loss} s^{c}$ & Loss cost of energy storage system in charge \\
\hline $\operatorname{loss}^{d}$ & Loss cost of energy storage system in discharge \\
\hline$w$ & Weight value in objective function \\
\hline$\alpha$ & Responsibility allocation coefficient \\
\hline Load & Demand in distribution system \\
\hline Load $^{\text {ave }}$ & Average demand in distribution system \\
\hline
\end{tabular}

\section{INTRODUCTION}

With the continuous development of distributed transactions, the issue of energy transactions has also received more and more attention. This is because when the use of distributed energy reaches a certain level, their electricity consumption behavior will have a nonnegligible impact on the grid [1]-[2]. Generally speaking, there is a great uncertainty in the energy transactions of electric vehicles (EVs). If EVs can be dispatched effectively, these energy of EVs will be met and even have a positive impact on the grid. According to many researches, the peak-cut [3]-[5], uncertainty palliation [6][8] and other advantages can be obtained through reasonable dispatch. Although these studies have brought good results to the power grid, these studies are based on centralized management and lack detailed modeling of individuals.

Peer-to-peer (P2P) may be a new method to solve the above problem. $\mathrm{P} 2 \mathrm{P}$ can promote transactions among fragmented individuals. In the $\mathrm{P} 2 \mathrm{P}$ transaction mechanism, auction is an excellent choice for EVs, because $\mathrm{N}$ buyers correspond to $\mathrm{M}$ sellers in $\mathrm{P} 2 \mathrm{P}$ transaction. Auction generally reflects the participants' valuation, electricity and other factors. References [9]-[12] introduce different styles of auctions, which are applied in transactions between EVs. Reference [9] proposes an incentivized auction to decrease the cost and encourage the participation of EVs. Reference [10] solves demand response by an online double auction, which is also effective in protecting participants' privacy. Reference [11] proposes a double auction combined with Blockchain. Reference [12] promotes the transactions between uncertain photovoltaic and EVs in the form of auctions. Reference [13] proposes a strategy to regulate the trading behavior among microgrids belonging to different owners, which is designed based on dynamic non-cooperative game theory.

Although these researches have proved their feasibility and superiority through their own experiments, there is 
still a lack of consideration in the tradeoff between full use of energy and social benefits. To solve this problem, the energy matching model proposed in this paper is mainly divided into two stages. In the first stage, EVs obtain more current or historical data, and make bidding for double auction. In the second phase, CSO will propose a fixed price superior to that of real-time market for some mismatched EVs, maximizing self-interest and social welfare.

The remainder of this paper is organized as follows. Double auction and smart match of energy matching models are proposed in Sections II and model linearization is introduced in Sections III. In Section IV, we will analyze numerically the effectiveness of the energy matching model. Conclusion and future work are summarized in Section V.

\section{PROBLEM Formulation}

In the previous auction research, the energy reuse of participants who fail in auction was often neglected. In this section, double auction and smart match, which form the energy matching model, will be described in detail.

\section{A. Double auction}

In this stage, some EVs enter the energy matching system under the trend of demand or interest. These participants act as buyer or seller. They need to bid for their own demand/supply, which is an incomplete information game. Thanks to blockchain technology, transaction data is in public. Therefore, participants can receive more current or historical information from the blockchain, which is helpful for participants to make decision for more revenue. It will be reflected in the following model.

The mechanism of double auction is shown in Fig. 1. Buyers make bidding based on demand and valuation, arranged from highest to lowest. Sellers make bidding based on supply and valuation, arranged from lowest to highest, as shown in Fig. 1 below:

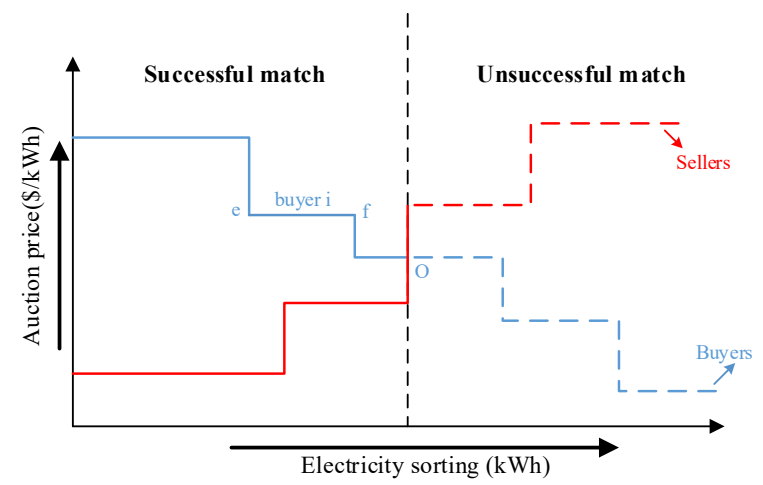

Fig.1: Schematic diagram of double auction

Buyer $i$ generally has its own valuation $v_{i}^{b}$, which is generally affected by power quality, demand urgency and so on. The buyer's rank in an auction is related to his valuation. Generally speaking, the higher the valuation, the more likely it will be ranked high. The relationship between rank and valuation is shown below:

$$
e\left(v_{i}^{b}\right)=\sum_{k=1, k \neq i}^{N u m} P\left(b_{k}>b_{i}\right) \cdot W_{k}^{b}-\frac{1}{2} \cdot W_{i}^{b}
$$

$$
f\left(v_{i}^{b}\right)=\sum_{k=1, k \neq i}^{N u m} P\left(b_{k}>b_{i}\right) \cdot W_{k}^{b}+\frac{1}{2} \cdot W_{i}^{b}
$$

Set $O$ as the auction breakpoint and $O$ as the projection of $O$ onto x-axis. By comparing the values $e, f$ and $o$, participants can determine their auction status (success or failure). Blockchain can present more historical data for participants to predict and judge the above values. Therefore, we can get the best bidding strategy of buyer $i$ by maximizing his revenue expectations.

$$
\begin{aligned}
b_{i}^{*} & =\arg \max \left\{P\left[f\left(v_{i}^{b}\right)<o\right] \cdot W_{i}^{b} \cdot\left(v_{i}^{b}-b_{i}\right)\right. \\
& +P\left[e\left(v_{i}^{b}\right)<o<f\left(v_{i}^{b}\right)\right] \cdot E^{b}\left(v_{i}^{b}\right) \cdot W_{i}^{b} \cdot\left(v_{i}^{b}-b_{i}\right)
\end{aligned}
$$

The best bidding strategy of seller $\mathrm{j}$ can be obtained in a similar way. Each participant will submit own best bidding, depending on the role they play.

\section{B. Smart match}

Consider that the transaction will stop after point $a$, as shown in Fig. 1. At this moment, CSO only needs to propose a price slightly better than the real-market clearing price, and EVs that do not successfully match will be willing to trade with CSO. After the transaction, CSO could either store the excess electricity or sell it to the grid or use the energy storage to meet the EV's demand for benefit.

First, we need to screen the EVs participating in the second stage and their willingness. Take the buyers as an example, we believe that if his valuation is lower than the real-market clearing price, he will not be able to participate in the second stage due to the inability to seek benefit. For other participants, they will have benefit to seek at this stage, and the benefit that can be sought will determine their willingness to participate. The willingness will be expressed in terms of the probability that it is willing to trade with CSO. It is expressed as follows:

$$
\operatorname{Pr}_{i, t}^{b}=\left\{\begin{array}{cc}
\frac{v_{i, t}^{b}-v^{b, C S O}}{\lambda_{i, t}^{b, v a l}-\varphi\left(v_{i, t}^{b}\right)} & \varphi\left(v_{i, t}^{b}\right)<b^{C S O} \\
1 & \varphi\left(v_{i, t}^{b}\right) \geq b^{C S O}
\end{array}\right.
$$

After screening EVs who participant in the second stage, we can build a mathematical model from the perspective of CSO. The objective function of CSO can contain two parts, that is, cost and social welfare.

$$
\min F=C+w \cdot \delta
$$

The cost is mainly divided into two parts, the purchase cost and the charge/discharge cost, which can be shown as follows: 


$$
\begin{aligned}
& C=\sum_{t=1}^{\operatorname{Nam}_{2} T}\left(\sum_{j=1}^{\operatorname{Nam} \_S} W_{j, t}^{s, C S O} \cdot s^{C S O} \cdot \operatorname{Pr}_{j, t}^{S}-\sum_{i=1}^{\operatorname{Nam}_{-} B} W_{i, t}^{b, C S O} \cdot b^{C S O} \cdot \operatorname{Pr}_{i, t}^{b}\right)
\end{aligned}
$$

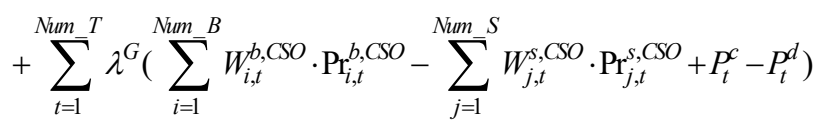

Social welfare is mainly reflected in the peak cut of load curve. Considering that a CSO has limited influence on the grid, a responsibility allocation coefficient $\alpha$ is proposed to divide peak cut tasks equally into CSOs. Therefore, CSO only needs to consider peak cut for $\alpha D$. Hence,

$$
\begin{aligned}
& \delta=\sum_{t=1}^{\text {Num }_{-} T} \mid \alpha \cdot \text { Load }_{t}-\sum_{i=1}^{\text {Num }^{B} B} W_{i, t}^{b, C S O} \cdot \operatorname{Pr}_{i, t}^{b} \\
& +\sum_{j=1}^{\text {Num }_{-} S} W_{j, t}^{s, C S O} \cdot \operatorname{Pr}_{j, t}^{s}-P_{t}^{c}+P_{t}^{d}-\text { Load }^{\text {ave }} \mid
\end{aligned}
$$

Where

$$
\begin{aligned}
& \text { Load }^{\text {ave }}=\frac{1}{N T} \sum_{t=1}^{\text {Num }}{ }^{T}\left(\alpha \cdot \text { Load }_{t}-\sum_{i=1}^{\text {Num }} B{ }^{B} W_{i, t}^{b, C S O} \cdot \operatorname{Pr}_{i, t}^{b}\right. \\
& \left.+\sum_{j=1}^{\text {Num }}{ }^{S} W_{j, t}^{s, C S O} \cdot \operatorname{Pr}_{j, t}^{s}-P_{t}^{c}+P_{t}^{d}\right)
\end{aligned}
$$

The corresponding constraints are briefly described below:

- Limits of battery capacity, charge and discharge behavior.

- Limits of transaction amount traded with EVs.

- Limits on the transaction amount between the CSO and the grid.

- Relationship on the transaction amount and participant willing.

After introducing the objective function and constraints of $\mathrm{CSO}$, the linearization of the above model will be performed in Section III.

\section{MODEL LINEARIZATION}

In the scheduling scheme introduced in Section II, the nonlinear term becomes a factor that weakens the computational efficiency. Moreover, this nonlinear term only exists in the absolute value part of Equation (9), so the main problem to be solved in Section III is to simplify the programming problem, namely, to linearize the absolute value part. In some cases, the absolute value term can be converted into a linear form, as shown in [14].

Equation (9) is expressed as the sum of multiple absolute values. Because (9) is nonlinear, it causes great inconvenience to the solution process. For the convenience of the solution process, the nonlinear part in (7) can be equivalent to the following form.

$$
\begin{gathered}
\min F=C+w \cdot \sum_{t=1}^{\text {Num }_{-}^{T}}\left(u_{t}^{+}+u_{t}^{-}\right) \\
\text {s.t. } \\
\alpha \cdot \text { Load }_{t}-\sum_{i=1}^{\text {Num } B}{ }_{i, t}^{b, C S O} \cdot \operatorname{Pr}_{i, t}^{b}+\sum_{j=1}^{\text {Num } S} W_{j, t}^{s, \text { CSO }} \cdot \operatorname{Pr}_{j, t}^{s} \\
-P_{t}^{c}+P_{t}^{d}-\text { Load }^{\text {ave }}=u_{t}^{+}-u_{t}^{-} \\
u_{t}^{+}, u_{t}^{-} \geq 0
\end{gathered}
$$

Other constraints

This equivalent method is from [14] and is applicable to the minimization of the absolute value part. The minimum function can effectively ensure that one of the auxiliary variables $u_{t}^{+}, u_{t}^{-}$must be 0 , so as to avoid the appearance of $u_{t}^{+} \cdot u_{t}^{-}$.

\section{CASE Studies}

In order to show the advantages of the energy matching mechanism proposed in this paper, we set up the following case to prove it.

In the case design, we mainly compared some indexes before and after the application of the energy matching mechanism, mainly including the impact on the power grid, the participants utility, and the variation of energy storage. The validity of the energy matching model is proved by utilizing the EV behavior data from [15]. 150 $\mathrm{EV}$ buyers and $100 \mathrm{EV}$ sellers are chosen to take part in energy matching mechanism. Fig. 2 shows the load curve that CSO needs to balance.

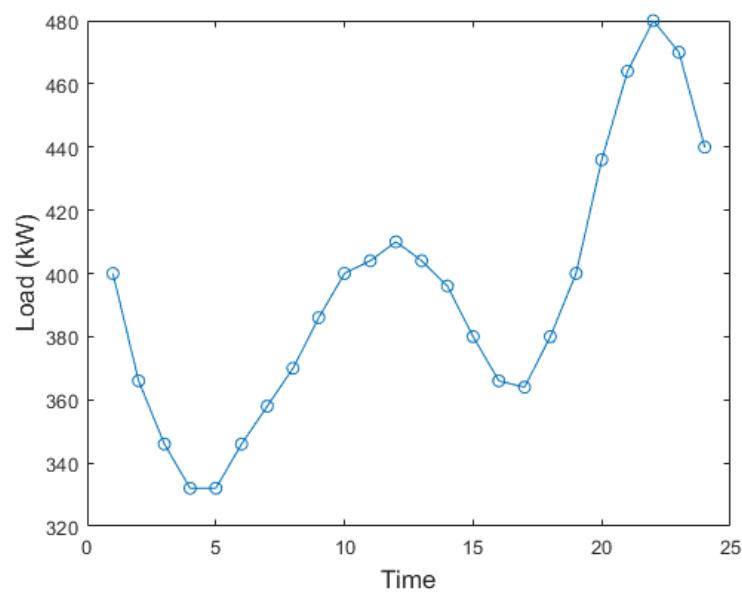

Fig. 2: Load curve that a CSO needs to balance

\section{A. The impact on the power grid}

In this part, the influence of the energy matching mechanism on the power grid will be highlighted. Fig. 3 compares the load curve in the grid before and after the application of the energy matching mechanism. 


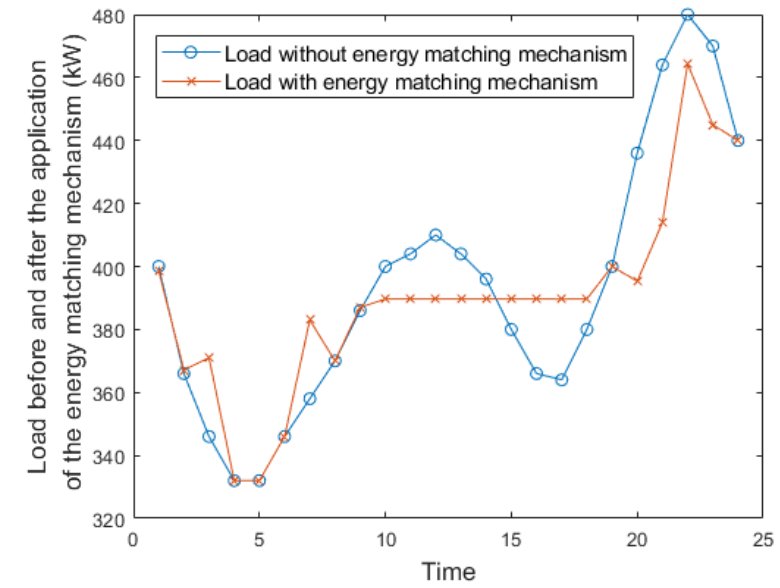

Fig. 3: Load curves before and after performing the energy matching mechanism

It is obvious that the load curve in the power grid will be improved to some extent after adding the energy matching mechanism. After smart match, the standard deviation of load curve is 30.16 , compared to the original 40.61 .

\section{B. The utility of participants}

This section compares the transaction difference before/after EVs participation in the energy match. Table I shows the cost/revenue of buyers/sellers when they choose to participate or not.

Table 1: Cost (Revenue) of buyers (sellers) before and after implementing the energy matching mechanism

\begin{tabular}{ccc}
\hline \multicolumn{3}{c}{ implementing the energy matching mechanism } \\
\hline Participant & Buyer & Seller \\
\hline $\begin{array}{c}\text { Total cost/revenue in } \\
\text { double auction }(\$)\end{array}$ & 86.52 & 86.52 \\
$\begin{array}{c}\text { Total cost/revenue in } \\
\text { smart match }(\$)\end{array}$ & 19.64 & 6.81 \\
$\begin{array}{c}\text { Total cost/revenue in } \\
\text { energy match process (\$) } \\
\text { Total cost/revenue } \\
\text { without energy match } \\
\text { process }(\$)\end{array}$ & 284.01 & 168.39 \\
\hline
\end{tabular}

As can be seen from Table 1, both the buyer's cost and the seller's revenue have been improved after participating in the energy matching mechanism. After the addition of the energy matching mechanism, the average cost of EV buyers decreased from $\$ 1.98$ to $\$ 1.89$, and the average income of EV sellers increased from $\$ 1.53$ to $\$ 1.68$. This proves that participants can benefit from this energy matching mechanism, which encourages more EVs to participate in this mechanism.

\section{The energy storage of CSO}

Under the energy matching mechanism, we can see the details of CSO's energy storage system, as shown in Fig. 4 below:

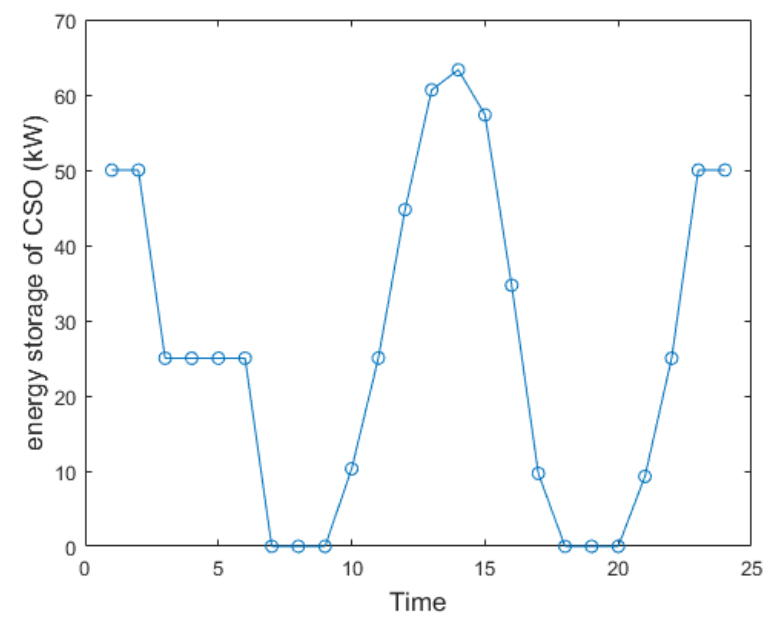

Fig. 4: The variation of CSO energy storage

From Fig. 4, it can be seen that the energy storage strategy of CSO will be related to the load curve to some extent. CSO discharges at the low point of the curve and charges at the peak of the curve. It is keeping with tendency of CSO behavior.

\section{CONCLUSION}

This paper presents a novel energy matching mechanism for CSO and EVs, mainly including double auction and smart match. Firstly, a new incomplete information game is proposed in the double auction. It is used in situations where current or historical information is more transparent, such as the blockchain. In a more transparent trading environment, participants can get more self-interested decisions to participate in the auction, which is reflected in the decrease of buyer average cost from $\$ 1.98$ to $\$ 1.89$, and the increase of seller average income from $\$ 1.53$ to $\$ 1.68$. Different from the traditional auction mechanism, smart match is added after the double auction, which is used for absorbing energy of EVs and cutting peak demand. Moreover, the standard deviation of the load curve is reduced from 40.61 to 30.16 . On the whole, the effectiveness mechanism has been proved. It not only encourages participants to pursue higher interests by introducing a transparent environment, but also facilitates energy consumption and peak cut.

In the future work, it will be a good choice to combine the power flow and voltage in the power system. For example, considering how transaction between EVs affects the stability of the grid, and a considerate planning would improve the operation.

\section{ACKNOWLEDGMENT}

This work was supported in part by the Department of Education of Guangdong Province: New and Integrated Energy System Theory and Technology Research Group under Grant 2016KCXTD022, in part by the Brunel Research Initiative and Enterprise Fund (BRIEF), in part by the National Natural Science Foundation of China under Grant 51907031, and in part by the Operation Fund of Guangdong Key Laboratory of Clean Energy Technology under Grant 2017B030314127. 
This article has been accepted for publication in a future issue of this conference proceedings, but has not been fully edited. Content may change prior to final publication. Citation information: DOl10.1109/PESA50370.2020.9344037, 2020 8th International Conference on Power Electronics Systems and Applications (PESA)

\section{REFERENCES}

[1] Zhao, Z., Yang, P., Xu, Z. and Guerrero, J. M.: 'Dynamic Characteristics Analysis and Stabilization of PV-Based Multiple Microgrid Clusters', IEEE Transactions on Smart Grid, 10, (1), Jan. 2019, pp. 805-818.

[2] Zhao, Z., Yang, P., Guerrero, J.M., Xu, Z. and Green, T.C.: 'Multiple-Time-Scales Hierarchical Frequency Stability Control Strategy of Medium-Voltage Isolated Microgrid', IEEE Transactions on Power Electronics, 31, (8), Aug. 2016, pp. 5974-5991.

[3] Dang, Q.: 'Electric vehicle (EV) charging management and relieve impacts in grids', 2018 9th IEEE International Symposium on Power Electronics for Distributed Generation Systems (PEDG), Charlotte, NC, 2018, pp. 1-5.

[4] Yamasaki, J., Tominaga, R., Ishii, Y., Shimizu, A., Kinoshita, S. and Wakao, S.: 'Optimization of installation and operation for retail store with photovoltaic, storage battery and EV quick charger', 2011 37th IEEE Photovoltaic Specialists Conference, Seattle, WA, 2011, pp. 001893-001896.

[5] Su, X., Li, W. and Li, X.: 'Research on ordered charging of battery swapping station based on adaptive genetic algorithm', 2019 IEEE Sustainable Power and Energy Conference (iSPEC), Beijing, China, 2019, pp. 792-796.

[6] Xu, N. Z. and Chung, C. Y.: 'Uncertainties of EV charging and effects on well-being analysis of generating systems', IEEE Transactions on Power Systems, 30, (5), Sept. 2015, pp. 2547-2557.

[7] Islam, M. R., Lu, H. H., Hossain, M. J. and Li, L.: 'Compensating neutral current, voltage unbalance and improving voltage of an unbalanced distribution grid connected with EV and renewable energy sources', 2019 22nd International Conference on Electrical Machines and Systems (ICEMS), Harbin, China, 2019, pp. 1-5.

[8] Ma, Y., Deng, F., Song, X. and Piao, C.: 'A fuzzy random scheduling model of islanded microgrid considering dual uncertainty of EV', 2020 Asia Energy and Electrical Engineering Symposium (AEEES), Chengdu, China, 2020, pp. 230-235.

[9] Zeng, M., Leng, S., Maharjan, S., Gjessing, S. and He, J.: 'An incentivized auction-based group-selling approach for demand response management in V2G systems', IEEE Transactions on Industrial Informatics, 11, (6), Dec. 2015, pp. 1554-1563.

[10] Li, D., Yang, Q., Yu, W., An, D., Zhang, Y. and Zhao, W.: 'Towards differential privacy-based online double auction for smart grid', IEEE Transactions on Information Forensics and Security, 15, 2020, pp. 971-986.

[11] Ping, J., Chen, S., Yan, Z., Wang, H., Yao, L. and Qian, M.: 'EV charging coordination via blockchain-based charging power quota trading', 2019 IEEE Innovative Smart Grid Technologies-Asia (ISGT Asia), Chengdu, China, 2019, pp. $4362-4367$.

[12] Kikusato, H., Fujimoto, Y., Hanada, S., Isogawa, D., Yoshizawa, S., Ohashi, H., Hayashi, Y.: 'Electric vehicle charging management using auction mechanism for reducing PV curtailment in distribution systems', IEEE Transactions on Sustainable Energy, 11, (3), July 2020, pp. 1394-1403.

[13] Zhao, Z., Guo, J., Lai, C. S., Xiao, H., Zhou, K. and Lai, L. L.: 'Distributed Model Predictive Control Strategy for Islands Multi-Microgrids Based on Non-Cooperative Game', IEEE Transactions on Industrial Informatics, to be published.

[14] Wang Y., Huang, L., Shahidehpour, M., Lai, L. L. and Zhou, Y.: 'Impact of cascading and common-cause outages on resilience-constrained optimal economic operation of power systems', IEEE Transactions on Smart Grid, 11, (1), Jan. 2020, pp. 590-601.
[15] Muratori M.: 'Impact of uncoordinated plug-in electric vehicle charging on residential power demand', Nature Energy, 3, 2018, pp. 193-201. 